\title{
A Linear Bound on the Diameter of the Transportation Polytope *
}

\author{
Graham Brightwell $^{\dagger} \quad$ Jan van den Heuvel ${ }^{\dagger} \quad$ Leen Stougie ${ }^{\ddagger}$
}

\begin{abstract}
We prove that the combinatorial diameter of the skeleton of the polytope of feasible solutions of any $m \times n$ transportation problem is at most $8(m+n-2)$.
\end{abstract}

The transportation problem ( TP ) is a classic problem in operations research. The problem was posed for the first time by Hitchcock in 1941 [9] and independently by Koopmans in 1947 [12], and appears in any standard introductory course on operations research.

The $m \times n$ TP has $m$ supply points and $n$ demand points. Each supply point $i$ holds a quantity $r_{i}>0$, and each demand point $j$ wants a quantity $c_{j}>0$, with $\sum_{i=1}^{m} r_{i}=\sum_{j=1}^{n} c_{j} . \mathrm{A}$ solution to the problem can be written as an $m \times n$ matrix $X$, where entries are decision variables $x_{i j}$ having value equal to the amount transported from supply point $i$ to demand point $j$. The set of feasible solutions of TP, the transportation polytope $\mathcal{T}$, is described by

$$
\begin{aligned}
\sum_{j=1}^{n} x_{i j}=r_{i}, & i=1,2, \ldots, m ; \\
\sum_{i=1}^{m} x_{i j}=c_{j}, & j=1,2, \ldots, n ; \\
x_{i j} \geq 0, & i=1,2, \ldots, m, j=1,2, \ldots n .
\end{aligned}
$$

The 1-skeleton (edge graph) of $\mathcal{T}$ is defined as the graph with vertices the vertices of the polytope and edges its 1-dimensional faces. The diameter of $\mathcal{T}$, which we denote by $\operatorname{diam}(\mathcal{T})$, is the diameter of its 1-skeleton. In 1957 W.M. Hirsch stated his famous conjecture ( cf. [5]) saying that any $d$-dimensional polytope with $n$ facets has diameter at most $n-d$. So far the best known bound for arbitrary polytopes is $O\left(n^{\log d+1}\right)$ [10]. Any polynomial bound is still lacking. Such bounds have been proved for some special classes of polytopes ( for examples, see [14]). Among those are some special classes of transportation polytopes $[1,3]$ and the polytope of the dual of TP [1].

\footnotetext{
${ }^{*}$ Research for this paper was done while the second and third author were visiting the Isaac Newton Institute for Mathematical Sciences, Cambridge, U.K. All authors were supported by the TMR Network DONET of the European Community ERB TMRX-CT98-0202.

${ }^{\dagger}$ Centre for Discrete and Applicable Mathematics and Department of Mathematics, London School of Economics, U.K., \{graham,jan\}@maths.lse.ac.uk.

${ }^{\ddagger}$ Technische Universiteit Eindhoven and CWI Amsterdam, The Netherlands, leen@win.tue.nl.
} 
The first polynomial bound on $\operatorname{diam}(\mathcal{T})$ was given by Dyer and Frieze [7]. Actually, they prove a bound on the diameter of any polytope $\left\{x \in \mathbb{R}^{n} \mid A x \leq b\right\}$, where $A$ is a totally unimodular matrix and $b \in \mathbb{R}^{m}$. The proof is complicated and indirect, using the probabilistic method, and the bound is huge $\left(O\left(m^{16} n^{3} \log ^{3} n\right)\right)$.

We will give a simple proof that $\operatorname{diam}(\mathcal{T}) \leq 8(m+n-2)$. The proof is constructive: it gives an algorithm that describes how to go from any vertex to any other vertex on $\mathcal{T}$ in at most $8(m+n-2)$ steps along the edges.

We first review some known facts about $\mathcal{T}$. A transportation polytope is degenerate if there exist $I \subset\{1, \ldots, m\}$ and $J \subset\{1, \ldots, n\}$, with $0<|I|+|J|<n+m$ and $\sum_{i \in I} r_{i}=\sum_{j \in J} c_{j}$. By [11, Theorem 5] we may concentrate on nondegenerate transportation polytopes by applying small perturbations on supplies and demands in case of degeneracy. The dimension of $\mathcal{T}$ is $m n-m-n+1$ [11] if $\mathcal{T}$ is nondegenerate. Thus, if the Hirsch Conjecture is true, $\operatorname{diam}(\mathcal{T}) \leq m+n-1$.

We denote by $K_{m, n}$ the complete bipartite graph with vertices representing the supply points of TP as one color class and vertices representing the demand points of TP as the other color class. For any feasible solution $X \in \mathcal{T}$, let $G(X)$ be the subgraph of $K_{m, n}$ with edge set $E(X)=\left\{(i, j) \mid x_{i j}>0, i=1, \ldots, m, j=1, \ldots, n\right\}$.

\section{Lemma 1 [11]}

Given a nondegenerate transportation polytope $\mathcal{T}$, a feasible solution $X$ is a vertex of $\mathcal{T}$ if and only if $G(X)$ is a spanning tree.

We explain a pivot operation ( step from one vertex on the 1-skeleton of $\mathcal{T}$ to a neighbouring vertex ) as an operation on the corresponding spanning trees. Given a vertex $X$, an edge $(a, b) \notin E(X)$ is inserted in $G(X)$, creating a unique cycle $C$. Since $C$ is an even cycle we can label its edges alternatingly + and - , starting with label + for $(a, b)$. Let $E^{+}(C)$ and $E^{-}(C)$ be the edges of $C$ with respectively label + and -, and let $(c, d)$ be the edge in $E^{-}(C)$ with $x_{c d}$ minimal. Removing $(c, d)$ from $G(X) \cup(a, b)$ finishes the pivot operation, which we call a pivot on $(a, b)$.

The above corresponds to increasing the value of all $x_{i j}$ with $(i, j) \in E^{+}(C)$ with the amount $x_{c d}$ and decreasing all $x_{i j}$ with $(i, j) \in E^{-}(C)$ with the same amount. In particular, $x_{a b}$ is raised from 0 to $x_{c d}$ (becomes nonzero variable), and $x_{c d}$ gets value 0 (becomes zero variable). Since we assumed nondegeneracy, no other variable corresponding to an edge in $E^{-}(C)$ becomes zero.

\section{Theorem 2}

For any pair $X, Y$ of vertices of an $m \times n$ transportation polytope, at most $8(m+n-2)$ pivot steps are required to go from $X$ to $Y$.

Proof. We use induction on $m+n$. If $m=1$ or $n=1$, then $K_{m, n}$ is the graph of the only feasible solution, and the theorem is trivially true. Now assume $m \geq 2$ and $n \geq 2$. The proof is based upon the following claim. 


\section{Claim}

For any two vertices $X$ and $Y$, there are vertices $X^{\prime}$ and $Y^{\prime}$ and points $a$ and $b$ such that $a$ is a leaf incident to $b$ in both $G\left(X^{\prime}\right)$ and $G\left(Y^{\prime}\right)$ and $X^{\prime}$ and $Y^{\prime}$ can be reached from, respectively, $X$ and $Y$ by a total of at most 8 pivot steps.

Suppose that the claim is true and $a$ is a supply point, whence $r_{a}<c_{b}$. We may remove $a$ from the problem and set the demand of $b$ to $c_{b}-r_{a}$. The new problem is again nondegenerate and the matrices $X^{\prime}$ and $Y^{\prime}$, with the row corresponding to $a$ deleted, are vertices for the new problem with $m+n-1$ supply and demand points. The theorem then follows by induction. A similar reasoning holds if $a$ is a demand point.

To prove the claim, we define, for each supply point $i$, demand point $j$, and vertex $Z$, $D_{i j}(Z)=d_{G(Z)}(i)+d_{G(Z)}(j)$, the sum of the degrees of $i$ and $j$ in $G(Z)$. We distinguish two cases.

CASE 1: There exist $a$ and $b$ with $D_{a b}(X)+D_{a b}(Y) \leq 8$.

Suppose without loss of generality that $a$ is a supply point and $r_{a}<c_{b}$. This implies that there is no vertex in which $(a, b)$ is the only edge incident to $b$.

If $(a, b) \in E(X)$ we set $X_{0}=X$. Otherwise we insert $(a, b)$ in a single pivot step, creating $X_{0}$. Thus, always $D_{a b}\left(X_{0}\right) \leq D_{a b}(X)+2$. If $(a, b)$ is the only edge incident to $a$ in $X_{0}$ then we choose $X^{\prime}=X_{0}$ and halt. Otherwise, there exist $j \neq b$ such that $(a, j) \in E\left(X_{0}\right)$ and, since $r_{a}<c_{b}$, there must also exist $i \neq a$ such that $(i, b) \in E\left(X_{0}\right)$. This gives a path $(j, a, b, i)$ of length three. By performing a pivot on $(i, j)$ we obtain a new vertex $X_{1}$. Because $(a, b)$ also received label + on cycle $(j, a, b, i, j)$, we have that $(a, b) \in E\left(X_{1}\right)$. Since $(a, j)$ and $(i, b)$ are the only edges with label - on $(j, a, b, i, j)$, one of them is deleted in the pivot step. Therefore $D_{a b}\left(X_{1}\right)=D_{a b}\left(X_{0}\right)-1$.

We repeat this procedure on consecutive vertices until arriving at a vertex $X^{\prime}$ with $d_{G\left(X^{\prime}\right)}(a)=1$. Since $d_{G\left(X^{\prime}\right)}(b) \geq 2\left(\right.$ as $\left.r_{a}<c_{b}\right)$, we have $D_{a b}\left(X^{\prime}\right) \geq 3$, and hence this occurs after at most $1+D_{a b}\left(X_{0}\right)-D_{a b}\left(X^{\prime}\right) \leq D_{a b}\left(X_{0}\right)-2 \leq D_{a b}(X)$ pivot steps, including the one to go from $X$ to $X_{0}$.

In the same way we go from $Y$ to $Y^{\prime}$ with $a$ a leaf adjacent to $b$ in $G\left(Y^{\prime}\right)$. Thus, the total number of pivot steps is at most $D_{a b}(X)+D_{a b}(Y)$, which proves the claim in this case.

CASE 2: For all $a$ and $b, D_{a b}(X)+D_{a b}(Y)>8$.

Suppose without loss of generality that $n \leq m$. We choose a supply point a minimizing $d_{G(X)}(a)+d_{G(Y)}(a)$ and, among those with the minimum value of $d_{G(X)}+d_{G(Y)}$, the point minimizing $r_{a}$. Since $|E(X)|+|E(Y)|=2 m+2 n-2$ we have $d_{G(X)}(a)+d_{G(Y)}(a) \leq$ $\lfloor(2 m+2 n-2) / m\rfloor \leq 3$. Hence, every demand point must have sum of degrees at least 6 , so $2 m+2 n-2 \geq 6 n$, implying that $m \geq 2 n+1$. In its turn this implies that $d_{G(X)}(a)+$ $d_{G(Y)}(a)=2$, in other words $a$ is already a leaf in both $G(X)$ and $G(Y)$. Let $A$ be the set of all supply points that are leaves in both trees. So $a \in A$ and, by choice of $a$, we have $r_{a} \leq r_{i}$ for all $i \in A$.

Consider the subtrees $G^{\prime}(X)$ and $G^{\prime}(Y)$ obtained by deleting all points in $A$. Since they contain $2(m-|A|)+2 n-2$ edges together, and since their supply points have total degree at least 3 , we have $3(m-|A|) \leq 2(m-|A|)+2 n-2$, implying that $m-|A| \leq 2 n-2$. Hence, there is a demand point $b$ with $d_{G^{\prime}(X)}(b)+d_{G^{\prime}(Y)}(b) \leq\lfloor(2(m-|A|)+2 n-2) / n\rfloor \leq\lfloor(6 n-6) / n\rfloor \leq 5$. As noted before, $d_{G(X)}(b)+d_{G(Y)}(b) \geq 6$. Thus, $b$ is adjacent to some point $i \in A$ in $G(X)$ or $G(Y)$. By the choice of $a$, this implies immediately that $r_{a} \leq r_{i}<c_{b}$. 
Below we will show that there exists a vertex $X^{\prime}$ in which $a$ is a leaf adjacent to $b$ and which is at most $1+d_{G^{\prime}(X)}(b)$ pivot steps away from $X$. By symmetry the same holds for $Y$. Since $d_{G^{\prime}(X)}(b)+d_{G^{\prime}(Y)}(b) \leq 5$, this proves the claim and hence the theorem.

Case 2.1: There is an $i \in A$ with $(i, b) \in E(X)$.

If $i=a$, no pivot step is needed: $X^{\prime}=X$. Otherwise, $(a, j) \in E(X)$ for some $j \neq b$. Insert $(a, b)$ in one pivot step. Notice that, apart from $a$, no point in $A$ is involved in this pivot step. In particular, $i$ is still a leaf adjacent to $b$ in the new vertex. If $(a, j)$ was deleted, we have reached our goal in one pivot step. Otherwise, inserting $(i, j)$ in a next pivot step will make $a$ a leaf adjacent to $b$, since by choice $r_{a} \leq r_{i}$. Therefore, at most two pivot steps are required to arrive at the desired $X^{\prime}$. Note that this is as required, since $G^{\prime}(X)$ is a tree with at least two demand points, so $2 \leq 1+d_{G^{\prime}(X)}(b)$.

CASE 2.2: There is no $i \in A$ with $(i, b) \in E(X)$.

Then $d_{G^{\prime}(X)}(b)=d_{G(X)}(b)>0$, and as in Case 1 we need $d_{G(X)}(b)+1$ pivot steps to get from $X$ to a suitable $X^{\prime}$, since $d_{G(X)}(a)=1$.

A direct consequence is :

\section{Corollary 3}

The transportation polytope has diameter at most $8(m+n-2)$.

The transportation problem is the problem of minimizing $\sum_{i=1}^{m} \sum_{j=1}^{n} t_{i j} x_{i j}$ over $\mathcal{T}$, where $t_{i j}$ is the unit transportation cost from supply point $i$ to demand point $j$. A polynomial, but not strongly polynomial, primal simplex algorithm has been presented in [13]. Here we use strongly polynomial to indicate that the bound on the running time is a polynomial function of $n$ and $m$ only, and not of the values in the vectors $r, c$. The existence of a strongly polynomial algorithm follows directly from [15]. This algorithm is not a primal simplex type algorithm. In fact the existence of a strongly polynomial primal simplex algorithm is unknown. It would be interesting to investigate if the result in this paper and its proof could help in the design of a strongly polynomial time primal simplex algorithm for the TP.

Special edges of the 1-skeleton of $\mathcal{T}$ are those that correspond to pivot steps in which the cycle $C$ used in the pivot operations has length four. We call these $2 \times 2$-pivot steps. In an earlier version of this paper [8] the authors derive a quadratic bound on the diameter of the 1-skeleton of the transportation polytope, in which the 1-skeleton is restricted to edges corresponding to $2 \times 2$-pivot steps only. This bound can be improved slightly, using the linear bound in the present paper, but remains quadratic. Such a bound is interesting for investigating if a random walk on the vertices of $\mathcal{T}$ using only $2 \times 2$-pivot operations mixes rapidly. The analysis of such a walk seems easier than one that allows steps along any edge of the polytope. This would be a crucial step in devising a polynomial randomized approximation scheme for counting the vertices of $\mathcal{T}$, a \#P-complete problem $[6]^{1}$. So far rapid mixing on $\mathcal{T}$ has been shown only for problems with a fixed number of rows or a fixed number of columns [4].

It remains open if $\operatorname{diam}(\mathcal{T}) \leq m+n-1$, as claimed by the Hirsch Conjecture. It is unlikely that our algorithm underlying the linear bound will, if subjected to a more subtle

\footnotetext{
${ }^{1}$ In fact, [6] only claims NP-hardness, but the proof establishes \#-completeness.
} 
analysis, lead to that result. The desired result requires, e.g., that between two vertices on $\mathcal{T}$ whose graphs have no edges in common, each of the pivot steps must decrease the difference between the two. This may suggest the stronger conjecture that for any two vertices that differ in $k$ nonzero variables there exists a pivot step that reduces the number of nonzero variables in which the vertices differ. We can prove that this is true if $k=1$, and it trivially holds if $k=m+n-1$. However, from an example on pages 141,142 of [2] we know that it is not true in general ( see [8] for details). A simpler example, pointed out by the referee, has two supply points with supply 3 each and three demand points with demand 2 each. The vertices $X$ and $Y$ with $x_{11}=x_{23}=y_{13}=y_{21}=2$ and $x_{12}=y_{12}=x_{22}=y_{22}=1$ differ in two nonzero variables. But there is no pivot step from $X$ that will result in a vertex $X^{\prime}$ which differs from $Y$ in only one nonzero variable, as can be checked easily.

Note that the example does not disprove the Hirsch Conjecture. (The graph of the transportation polytope corresponding to the example is a cycle with six vertices, hence has diameter 3, which is $m+n-1$ for $m=2, n=3$.) But it shows that pivot steps need to be chosen carefully to obtain the result.

Acknowledgement: We gratefully acknowledge discussions with Martin Dyer. We also thank the anonymous referee for suggestions that improved the presentation of this note.

\section{References}

[1] M. L. Balinski, On two special classes of transportation polytopes, Mathematical Programming Studies 1, 1974, 43-58.

[2] M. L. Balinski and F. J. Rispoli, Signature classes of transportation polytopes, Mathematical Programming 60, 1993, 127-144.

[3] E. D. Bolker, Transportation polytopes, Journal of Combinatorial Theory B 13, 1972, 251-262.

[4] M. Cryan, M. Dyer, H. Müller and L. Stougie, Random walks on the vertices of transportation polytopes with constant number of sources, Proceedings of the Fourteenth Annual ACM-SIAM Symposium on Discrete Algorithms (SODA), Baltimore, Maryland, 2003, 330-339.

[5] G. B. Dantzig, Linear programming and extensions, Princeton University Press, Princeton, New Jersey, 1963.

[6] M.E. Dyer, The complexity of vertex enumeration methods, Mathematics of Operations Research 8, 1983, 381-402.

[7] M. E. Dyer and A. M. Frieze, Random walks, totally unimodular matrices and a randomized dual simplex method, Mathematical Programming 64, 1994, 1-16.

[8] J. van den Heuvel and L. Stougie, A quadratic bound on the diameter of the transportation polytope, SPOR-report 2002-17, Technische Universiteit Eindhoven, and CDAM Research Report 2002-09, London School of Economics, 2002. Available on-line at http://www.cdam.lse.ac.uk/Reports/Files/cdam-2002-09.pdf 
[9] F. L. Hitchcock, The distribution of a product from several sources to numerous localities, Journal of Mathematics and Physics 20, 1941, 224-230.

[10] G. Kalai and D. J. Kleitman, A quasi-polynomial bound for the diameter of graphs of polyhedra, Bulletin of the American Mathematical Society 26, 1992, 315-316.

[11] V. Klee and C. Witzgall, Facets and vertices of transportation polytopes, in G. B. Dantzig and A.F. Veinott (eds.), Mathematics of the decision sciences, Part 1, American Mathematical Society, Providence, Richmond, 1968, 257-282.

[12] Tj. C. Koopmans, Optimum utilization of the transportation system, in D. H. Leavens (ed.), The Econometric Society Meeting, Washington DC, 1947, 1948, 136-146.

[13] J. B. Orlin, A polynomial time primal network simplex algorithm for minimum cost flows, Mathematical Programming 78, 1997, 109-129.

[14] A. Schrijver, Polyhedral combinatorics, in R. Graham, M. Grötschel and L. Lovász (eds.), Handbook of combinatorics, Volume II, North-Holland Elsevier, 1995, 1649-1704.

[15] E. Tardos, A strongly polynomial minimum cost circulation algorithm, Combinatorica 5, $1985,247-255$. 